\title{
Invariant Tensors in Gauge Theories
}

\author{
Dillon Berger, ${ }^{1}$ Jessica Howard, ${ }^{1}$ and Arvind Rajaraman, ${ }^{1}$ \\ ${ }^{1}$ Department of Physics and Astronomy, University of California, Irvine, CA 92697-4575 USA
}

\begin{abstract}
Invariant tensors play an important role in gauge theories, for example, in dualities of $N=1$ gauge theories. However, for theories with fields in representations larger than the fundamental, the full set of invariant tensors is often difficult to construct. We present a new approach to the construction of these tensors, and use it to find the complete set of invariant tensors of a theory of $S O(3)$ with fields in the symmetric tensor representation.
\end{abstract}

Invariant tensors, Gauge Theories

DOI: 10.31526/LHEP.2.2018.04

The invariant objects in a gauge theory play an important role. In a gauge theory, fields transform under various representations of the symmetry. However, the observables are gauge invariant and are hence invariant combinations of the fields. Similarly, in a confining theory, the physical spectrum is composed of gauge-invariant bound states of the fundamental fields.

Similarly, the duality of supersymmetric gauge theories was initiated by [1]; here two or more supersymmetric gauge theories were conjectured to be equivalent at low energies. Testing these duality conjectures requires detailed knowledge of the theory being studied. This is required for matching the flat directions, matching the spectra or the chiral rings, or for checking 't Hooft's anomaly matching conditions [2]. For all these tests, a knowledge of the gauge invariant operators is necessary. Indeed, one might expect an exact equivalence of the operators in these theories [3, 4, 5].

The invariant tensors of the fundamental representations of the classical groups are known ${ }^{1}$. However, the tensors for the other representations are not completely classified. For the exceptional groups such as $E_{6}$, it is not even known if the full set of tensors for the fundamental representation has been found [18].

Our goal in this paper is to present an approach to finding the fundamental polynomial invariants of gauge theories (this is sometimes known as a Hilbert basis). The elements of the Hilbert basis are said to be (polynomially) independent. We wish to find the invariant tensors for physically relevant groups and representations, with the goal of eventually being able to classify the tensors for the various representations of the exceptional groups.

Our approach is to successively reduce the symmetry group to a tractable subgroup for which the invariant structure is known. We then find the necessary invariants in the full theory that are required to reproduce the invariants of the subgroup. This then yields all invariants of the larger group.

The method is more easily described by example. We begin by applying it to two simple cases for which the answer is known. These are the group $S O(2)$ with fields in the fundamental representation, and the group $S O(3)$ with fields in the fundamental. The invariants of these theories are well known, and we derive them by our method below.

${ }^{1}$ For a section of the mathematical literature relevant to invariant theory: [6]-
Finally, we apply our method to a nontrivial case: $S O(3)$ with fields in the symmetric tensor representation. The invariants here are not well known. However, we are able to prove that there are invariants of degree 2, 3, 4 and 5, and no others. We are also able to explicitly find the form of the invariants.

$S O(2)$ is the group of 2 by 2 orthogonal matrices (we are here using the physicist approach to groups and algebras). A general element can be represented as

$$
g=\left(\begin{array}{cc}
\cos \theta & \sin \theta \\
-\sin \theta & \cos \theta
\end{array}\right)
$$

where $0 \leq \theta<2 \pi$. The corresponding algebra is generated by the single matrix

$$
m=\left(\begin{array}{cc}
0 & -i \\
i & 0
\end{array}\right) .
$$

The general group element is related to the algebra element as $g=e^{i \theta m}$.

The fundamental representation can be written as a column vector of two elements

$$
r=\left(\begin{array}{l}
a \\
b
\end{array}\right)
$$

(One could also impose a condition on the norm e.g. $a^{2}+b^{2}=$ 1 , but this is not necessary).

The group acts on the representation as

$$
r \rightarrow g r,
$$

while the infinitesimal transformations act on the representation as

$$
r \rightarrow(1+i \theta m) r
$$

The $S O(2)$ algebra is isomorphic to a $U(1)$ algebra. If we define $\Phi=a+i b$, then the action of the group is just $\Phi \rightarrow \Phi e^{i \theta}$. If there are two different fields $\Phi_{1}, \Phi_{2}$, then the invariants are of the form $\operatorname{Re}\left(\Phi_{1} \Phi_{2}^{*}\right)$ or $\operatorname{Im}\left(\Phi_{1} \Phi_{2}^{*}\right)$ (or products thereof). These can be written in $S O(2)$ language as $r_{i} r_{i}^{\prime}$ or $\epsilon_{i j} r_{i} r_{j}^{\prime}$.

There are thus two invariants of degree 2 in this theory. As we have seen, this is easy to show directly. Nevertheless, we derive this using a more generalizable method.

Accordingly, we take the approach where we try to reduce the symmetry. We do this by choosing one particular fundamental and giving it a fixed value (i.e. what is called spontaneous symmetry breaking in quantum field theory). We choose the fundamental $r^{(0)}$ and set

$$
r^{(0)}=\left(\begin{array}{l}
0 \\
C
\end{array}\right)
$$


where $C$ is a constant. We will refer to this fixed value as the vev (short for vacuum expectation value).

This vev is fixed; therefore, the remaining symmetries are those that leave the vev fixed. In this case, there are no remaining symmetries. The vev has broken the symmetry from $S O(2)$ to nothing non-trivial.

Now consider a second fundamental

$$
r^{(1)}=\left(\begin{array}{l}
c \\
d
\end{array}\right) \text {. }
$$

Now the symmetry is completely broken; and hence the $S O(2)$ structure has no meaning. This is really two independent fields $c$ and $d$ with no symmetry linking them. The invariants are therefore immediate; they are any combinations of $c, d$. Hence when the symmetry is broken, the invariants are of degree 1 and are arbitrary combinations of the fields.

What does this tell us about the original theory, assuming that we knew nothing of the invariants of $S O(2)$ ?

We must have that the invariants of $S O(2)$ are such that after $r^{(0)}$ gets its fixed vev, they reduce to the degree 1 invariants of the broken theory.

The simplest way this could happen is if there were degree 1 invariants in the $S O(2)$ theory; it is easy to check that there are not.

The next possibility is a degree 2 invariant such as $r_{i}^{(0)} r_{i}^{(1)}$ which would become a degree 1 invariant once $r^{(0)}$ is replaced by a fixed value. Indeed, this is an invariant, and becomes $C d$ once evaluated. Here $C$ is just a constant, and is not relevant for the purposes of matching invariants.

We still need something that will give us the field $c$. This is the other degree 2 invariant $\epsilon_{i j} r_{i}^{(0)} r_{j}^{(1)}$ which becomes $-C c$.

These two invariants $r_{i}^{(0)} r_{i}^{(1)}, \epsilon_{i j} r_{i}^{(0)} r_{j}^{(1)}$ are therefore capable of generating all invariants of the broken theory.

We can therefore assert that $r_{i}^{(a)} r_{i}^{(b)}, \epsilon_{i j} r_{i}^{(a)} r_{j}^{(b)}$ generate the invariants of the full $S O(2)$ theory, where $r_{i}^{(a)}$ and $r_{j}^{(b)}$ are arbitrary fundamentals.

$S O(3)$ is the group of 3 by 3 orthogonal matrices. The corresponding algebra is generated by the three matrices

$$
\begin{array}{cc}
m_{1}=\left(\begin{array}{ccc}
0 & -i & 0 \\
i & 0 & 0 \\
0 & 0 & 0
\end{array}\right) & m_{2}=\left(\begin{array}{ccc}
0 & 0 & -i \\
0 & 0 & 0 \\
i & 0 & 0
\end{array}\right) \\
m_{3}=\left(\begin{array}{ccc}
0 & 0 & 0 \\
0 & 0 & -i \\
0 & i & 0
\end{array}\right) .
\end{array}
$$

The fundamental representation can be written as a column vector of three real elements

$$
r=\left(\begin{array}{l}
a \\
b \\
c
\end{array}\right) .
$$

The infinitesimal transformations act on the representation as

$$
r \rightarrow\left(1+i \sum_{i} \alpha_{i} m_{i}\right) r
$$

Again we try to reduce the symmetry. We do this by choosing one particular fundamental and giving it a fixed value. We choose the fundamental $r^{(0)}$ and give it a fixed vev

$$
r^{(0)}=\left(\begin{array}{l}
0 \\
0 \\
C
\end{array}\right)
$$

The remaining symmetries are those that leave the vev fixed. In this case, this would be a choice of $\alpha_{i}$ such that

$$
r^{(0)} \rightarrow\left(1+i \sum_{i} \alpha_{i} m_{i}\right) r^{(0)}=r^{(0)} .
$$

It is fairly straightforward to go through the exercise and show that the only possibility is $\alpha_{2}=\alpha_{3}=0$, while $\alpha_{1}$ is anything. This is a residual $S O(2)$ symmetry. The vev has broken the symmetry from $S O(3)$ to $S O(2)$.

Now consider a second fundamental

$$
r^{(1)}=\left(\begin{array}{l}
d \\
e \\
f
\end{array}\right)
$$

$r^{(1)}$ is in a reducible representation of $S O(2)$; in fact the $d$ and $e$ fields combine to form a fundamental of $S O(2)$, while $f$ is a singlet of $S O(2)$.

There are degree 2 invariants involving the fundamental, as described in the previous section, as well as degree 1 invariants from the singlets. More precisely, if we introduce a further fundamental

$$
r^{(2)}=\left(\begin{array}{l}
g \\
h \\
k
\end{array}\right)
$$

the invariants would be generated by the singlets $f, k$ the bilinear $(d g+e h)$ and the bilinear $(d h-g f)$.

It must be that the invariants of $\mathrm{SO}(3)$ are such that after $r^{(0)}$ gets its fixed vev, they reduce to these invariants.

A quick check shows that we need a bilinear invariant $r_{i}^{(a)} r_{i}^{(b)}$ and a trilinear invariant $\epsilon_{i j k} r_{i}^{(a)} r_{j}^{(b)} r_{k}^{(c)}$. (One needs to check that these are actually invariants, but that is also well known.) Specifically, the bilinears $r_{i}^{(0)} r_{i}^{(1)}, r_{i}^{(0)} r_{i}^{(2)}$ generate the degree 1 invariants after $r^{(0)}$ gets a vev, the bilinear $r_{i}^{(1)} r_{i}^{(2)}$ generates the bilinear $(\mathrm{dg}+\mathrm{eh})$, and the trilinear $\epsilon_{i j k} r_{i}^{(0)} r_{j}^{(1)} r_{k}^{(2)}$ generates the bilinear (dh-gf).

We can therefore assert that $r_{i}^{(a)} r_{i}^{(b)}, \epsilon_{i j k} r_{i}^{(a)} r_{j}^{(b)} r_{j}^{(c)}$ generate the invariants of the full $S O(3)$ theory with fundamentals.

The symmetric tensor representation can be written as a tensor $T_{i j}$ where $T_{i j}=T_{j i}$ and $\delta^{i j} T_{i j}=0$.

$$
T_{i j}=\left(\begin{array}{ccc}
T_{11} & T_{12} & T_{13} \\
T_{12} & T_{22} & T_{23} \\
T_{13} & T_{23} & -T_{11}-T_{22}
\end{array}\right)
$$
as

The infinitesimal transformations act on the representation

$$
T_{i j} \rightarrow T_{i j}+i\left(\sum_{a} \alpha_{a} m_{a}\right)_{i k} T_{k j}+i\left(\sum_{a} \alpha_{a} m_{a}\right)_{j k} T_{i k}
$$


We are looking for invariants; in this case, in fact, there is no answer in the literature for the full set of invariants. Note that we can contract the indices either using the invariant tensor $\delta_{i j}$ or the invariant tensor $\epsilon_{i j k}$. However, since an element of the symmetric tensor representation has an even number of indices, there must be an even number of epsilon tensors, tr which can then be converted to delta tensors. It follows, then, that we may write a general invariant of this representation as the trace of an arbitrary (matrix) product of symmetric tensors. We will denote such an invariant as $\operatorname{tr}(A B \ldots N)=$ $A_{i j} B_{k l} \ldots N_{m n} \delta_{j k} \delta_{l m} \delta_{n i}$.

Again we try to reduce the symmetry. We choose one fundamental $T^{(0)}$ and give it a fixed vev. However, there are many possible choices which are physically inequivalent. We will choose

$$
T_{11}^{(0)}=1 \quad T_{22}^{(0)}=1 \quad T_{33}^{(0)}=-2
$$

That is,

$$
T_{i j}^{(0)}=\left(\begin{array}{ccc}
1 & 0 & 0 \\
0 & 1 & 0 \\
0 & 0 & -2
\end{array}\right)
$$

This breaks the $S O(3)$ symmetry; the remaining continuous symmetry is the $S O(2)$ symmetry where $\alpha_{1}$ is nonzero, and the rest zero. Any other tensor $T_{i j}$ then decomposes into 5 fields, of charges $2,1,0,-1,-2$. The fields (with the charge labelled as a subscript) are explicitly:

$$
\begin{aligned}
T_{2} & =2 T_{12}+i\left(T_{11}-T_{22}\right) \\
T_{-2} & =T_{2}^{*} \\
T_{1} & =\left(\frac{i}{\sqrt{2}}-\frac{1}{\sqrt{2}}\right)\left(T_{13}-i T_{23}\right) \\
T_{-1} & =T_{1}^{*} \\
T_{0} & =T_{11}+T_{22}
\end{aligned}
$$

and so may be written as

$$
T_{i j}=\left(\begin{array}{ccc}
\frac{T_{0}}{2}+\frac{i T_{-2}}{4}-\frac{i T_{2}}{4} & \frac{T_{-2}}{4}+\frac{T_{2}}{4} & \frac{i T_{-1}}{2}-\frac{i T_{1}}{2} \\
\frac{T_{-2}}{4}+\frac{T_{2}}{4} & \frac{T_{0}}{2}+\frac{T_{2} i}{4}-\frac{i T_{-2}}{4} & \frac{T_{-1}}{2}+\frac{T_{1}}{2} \\
\frac{i T_{-1}}{2}-\frac{i T_{1}}{2} & \frac{T_{-1}}{2}+\frac{T_{1}}{2} & -T_{0}
\end{array}\right) .
$$

In addition, there is a discrete $Z_{2}$ symmetry which interchanges all indices 1 with an index of 2 , so that for example $T_{13} \leftrightarrow T_{23}$. This symmetry removes the epsilon tensor of the $S O(2)$ symmetry. Under the discrete symmetry which interchanges the 1 and 2 indices, we have, $T_{2} \leftrightarrow T_{-2}, T_{1} \leftrightarrow$ $T_{-1}, T_{0} \leftrightarrow T_{0}$

We now want to construct all possible combinations of these fields which are invariant under both the $U(1)$ and $Z_{2}$ symmetries.

For the $U(1)$, the invariants combinations are those where the charges sum to zero. These are $T_{2} T_{-2}^{\prime}, T_{1} T_{-1}^{\prime}, T_{2} T_{-1}^{\prime} \tilde{T}_{-1}$, $T_{-2} T_{1}^{\prime} \tilde{T}_{1}$ and $T_{0}$.

However, these are not all invariant under the $Z_{2}$ symmetry. Under the symmetry, for example, $T_{2} T_{-2}^{\prime} \rightarrow T_{2}^{\prime} T_{-2}$, so there are two linear combinations: $T_{2} T_{-2}^{\prime}+T_{2}^{\prime} T_{-2}$, which is invariant under $Z_{2}$, and $T_{2} T_{-2}^{\prime}-T_{2}^{\prime} T_{-2}$, which picks up a minus sign under a $Z_{2}$ transformation. The invariants are, then, either even or odd under the $Z_{2}$.

We then find that the following expressions are invariant under both the $U(1)$ and the $Z_{2}$ symmetries

$$
\begin{aligned}
& O_{1}=T_{0} \\
& O_{2}=\operatorname{Re}\left(T_{2} T_{-2}^{\prime}\right) \equiv T_{2} T_{-2}^{\prime}+T_{2}^{\prime} T_{-2} \\
& O_{3}=\operatorname{Re}\left(T_{1} T_{-1}^{\prime}\right) \equiv T_{1} T_{-1}^{\prime}+T_{1}^{\prime} T_{-1} \\
& O_{4}=\operatorname{Re}\left(T_{2} T_{-1}^{\prime} \tilde{T}_{-1}\right) \equiv T_{2} T_{-1}^{\prime} \tilde{T}_{-1}+T_{-2} T_{1}^{\prime} \tilde{T}_{1}
\end{aligned}
$$

On the other hand, there are combinations which are invariant under the $U(1)$, but odd under the $Z_{2}$, which are

$$
\begin{aligned}
\operatorname{Im}\left(T_{2} T_{-2}^{\prime}\right) & \equiv T_{2} T_{-2}^{\prime}-T_{2}^{\prime} T_{-2} \\
\operatorname{Im}\left(T_{1} T_{-1}^{\prime}\right) & \equiv T_{1} T_{-1}^{\prime}-T_{1}^{\prime} T_{-1} \\
\operatorname{Im}\left(T_{2} T_{-1}^{\prime} \tilde{T}_{-1}\right) & \equiv T_{2} T_{-1}^{\prime} \tilde{T}_{-1}-T_{-2} T_{1}^{\prime} \tilde{T}_{1}
\end{aligned}
$$

We can find new invariants by taking pairwise products of these. There are 6 possible pairwise products

$\begin{array}{ll}\text { (i) } \operatorname{Im}\left(T_{1} T_{-1}^{\prime}\right) \operatorname{Im}\left(T_{1} T_{-1}^{\prime}\right) & \text { (ii) } \operatorname{Im}\left(T_{1} T_{-1}^{\prime}\right) \operatorname{Im}\left(T_{2} T_{-2}^{\prime}\right)\end{array}$

(iii) $\operatorname{Im}\left(T_{1} T_{-1}^{\prime}\right)\left(T_{2} T_{-1}^{\prime} \tilde{T}_{-1}-T_{-2} T_{1}^{\prime} \tilde{T}_{1}\right)$

(iv) $\operatorname{Im}\left(T_{2} T_{-2}^{\prime}\right) \operatorname{Im}\left(T_{2} T_{-2}^{\prime}\right)$

(v) $\operatorname{Im}\left(T_{2} T_{-2}^{\prime}\right)\left(T_{2} T_{-1}^{\prime} \tilde{T}_{-1}-T_{-2} T_{1}^{\prime} \tilde{T}_{1}\right)$

(vi) $\left(T_{2} T_{-1}^{\prime} \tilde{T}_{-1}-T_{-2} T_{1}^{\prime} \tilde{T}_{1}\right)\left(T_{2} T_{-1}^{\prime} \tilde{T}_{-1}-T_{-2} T_{1}^{\prime} \tilde{T}_{1}\right)$.

In principle, all these are further invariants. However, many of these can be reexpressed in terms of the previously found $O_{1-4}$. In fact,

$$
\begin{aligned}
& \operatorname{Im}\left(T_{2} T_{-2}^{\prime}\right) \operatorname{Im}\left(T_{2} T_{-2}^{\prime}\right)=\operatorname{Re}\left(T_{2} T_{-2}^{\prime}\right) \operatorname{Re}\left(T_{2} T_{-2}^{\prime}\right) \\
& \quad-\operatorname{Re}\left(T_{2} T_{-2}\right) \operatorname{Re}\left(T_{2}^{\prime} T_{-2}^{\prime}\right) \\
& \operatorname{Im}\left(T_{1} T_{-1}^{\prime}\right) \operatorname{Im}\left(T_{1} T_{-1}^{\prime}\right)=\operatorname{Re}\left(T_{1} T_{-1}^{\prime}\right) \operatorname{Re}\left(T_{1} T_{-1}^{\prime}\right) \\
& \quad-\operatorname{Re}\left(T_{1} T_{-1}\right) \operatorname{Re}\left(T_{1}^{\prime} T_{-1}^{\prime}\right) \\
& \quad \operatorname{Im}\left(T_{2} T_{-2}^{\prime}\right) \operatorname{Im}\left(T_{2} T_{-1}^{\prime} \tilde{T}_{-1}\right)=\operatorname{Re}\left(T_{2} T_{-2}^{\prime}\right) \operatorname{Re}\left(T_{2} T_{-1}^{\prime} \tilde{T}_{-1}\right) \\
& \quad-\operatorname{Re}\left(T_{2} T_{-2}\right) \operatorname{Re}\left(T_{2}^{\prime} T_{-1}^{\prime} \tilde{T}_{-1}\right) \\
& \operatorname{Im}\left(T_{1} T_{-1}^{\prime}\right) \operatorname{Im}\left(T_{2} T_{-1}^{\prime} \tilde{T}_{-1}\right)=\operatorname{Re}\left(T_{1} T_{-1}^{\prime}\right) \operatorname{Re}\left(T_{2} T_{-1}^{\prime} \tilde{T}_{-1}\right) \\
& \quad-\operatorname{Re}\left(T_{1}^{\prime} T_{-1}^{\prime}\right) \operatorname{Re}\left(T_{2} T_{-1} \tilde{T}_{-1}\right) \\
& \quad+\operatorname{Re}\left(T_{1} \tilde{T}_{-1}\right) \operatorname{Re}\left(T_{2} T_{-1}^{\prime} T_{-1}^{\prime}\right) \\
& \quad-\operatorname{Re}\left(T_{1}^{\prime} \tilde{T}_{-1}\right) \operatorname{Re}\left(T_{2} T_{-1} T_{-1}^{\prime}\right) \\
& \operatorname{Im}\left(T_{2} T_{-1}^{\prime} \tilde{T}_{-1}\right) \operatorname{Im}\left(T_{2} T_{-1}^{\prime} \tilde{T}_{-1}\right)=\operatorname{Re}\left(T_{2} T_{-1}^{\prime} \tilde{T}_{-1}\right) \operatorname{Re}\left(T_{2} T_{-1}^{\prime} \tilde{T}_{-1}\right) \\
& \quad+\frac{1}{2} \operatorname{Re}\left(T_{2} T_{-2}\right) \operatorname{Re}\left(T_{-1}^{\prime} \tilde{T}_{1}\right) \operatorname{Re}\left(T_{-1}^{\prime} \tilde{T}_{1}\right) \\
& \quad-\frac{1}{2} \operatorname{Re}\left(T_{2} T_{-2}\right) \operatorname{Re}\left(T_{-1}^{\prime} \tilde{T}_{1}\right) \operatorname{Re}\left(T_{-1}^{\prime} \tilde{T}_{1}\right) \\
& \quad-\frac{1}{2} \operatorname{Re}\left(T_{2} T_{-2}\right) \operatorname{Re}\left(T_{-1}^{\prime} T_{1}^{\prime}\right) \operatorname{Re}\left(\tilde{T}_{-1} \tilde{T}_{1}\right) .
\end{aligned}
$$

However, the operator

$$
\mathrm{O}_{5}=\operatorname{Im}\left(T_{2} T_{-2}^{\prime}\right) \operatorname{Im}\left(T_{1} T_{-1}^{\prime}\right)
$$

cannot be expressed in terms of the $O_{1-4}$. The ring of operators is therefore generated by the operators $\mathrm{O}_{1-5}$.

We would now like to use these operators to pick from the sea of possibilities the unique $S O(3)$ invariant operators that allow us to generate the full space of $S O(3)$ invariants. So now, the question is whether we can generate all these invariants of the broken theory from $S O(3)$ invariant combinations of tensors when one $S O(3)$ tensor, which will be denoted $T^{(0)}$, gets a vev as in equation 16 . 

ants.

We start with the simplest possibilities for the $S O(3)$ invari-

No $S O(3)$ invariant of degree 1 exists (i.e. $\operatorname{tr}(T)=0$ for symmetric tensors).

There is one $S O(3)$ invariant of degree 2 i.e. the trace $\operatorname{tr}(A B)$, where $\mathrm{A}, \mathrm{B}$ are tensors. It is convenient to write this trace in terms of the $S O(2)$ fields. From the correspondence 17, we have

$\operatorname{tr}(A B)=\left(A_{-1} B_{1}+A_{1} B_{-1}\right)+\frac{1}{4}\left(A_{-2} B_{2}+A_{2} B_{-2}\right)+\frac{3 A_{0} B_{0}}{2}$.

If one of these tensors obtains a vev, i.e. $B=T^{(0)}$, this is

$$
\operatorname{tr}\left(A T^{(0)}\right)=3 A_{0}=3 O_{1} .
$$

We then conclude $\operatorname{tr}(A B)$ must be part of the chiral ring of $S O(3)$, and that the operator $O_{1}$ is generated from this element of the ring when a tensor gets a vev and breaks the group to $S O(2)$. That is,

$$
\mathrm{O}_{1}=\frac{1}{3} \operatorname{tr}\left(A T^{(0)}\right)
$$

The element $\operatorname{tr}(A B)$ also yields a $S O(2)$ bilinear when neither obtains a vev. This can be written as

$$
\operatorname{Re}\left(A_{1} B_{-1}\right)+\frac{1}{4} \operatorname{Re}\left(A_{2} B_{-2}\right)=\operatorname{tr}(A B)-\frac{3 A_{0} B_{0}}{2} .
$$

Thus, the specific combination of bilinears $\operatorname{Re}\left(A_{1} B_{-1}\right)+$ $\frac{1}{4} \operatorname{Re}\left(A_{2} B_{-2}\right)$ can also be generated by combinations of the bilinear element of the $S O(3)$ chiral ring. However, the other linear combinations of the bilinears cannot be produced by using the $S O(3)$ bilinear. We need to use another element of the $S O(3)$ chiral ring.

The next possibility is the invariant of degree 3: $\operatorname{tr}(A B C)$. Using the decomposition 17, we find

$$
\begin{aligned}
\operatorname{tr}(A B C) & =-\frac{A_{-1} B_{-1} C_{2}}{4}-\frac{A_{-1} B_{0} C_{1}}{4}-\frac{A_{-1} B_{1} C_{0}}{4}-\frac{A_{-1} B_{2} C_{-1}}{4} \\
& +\frac{A_{-2} B_{0} C_{2}}{8}-\frac{A_{-2} B_{1} C_{1}}{4}+\frac{A_{-2} B_{2} C_{0}}{8} \\
& -\frac{A_{0} B_{-1} C_{1}}{4}+\frac{A_{0} B_{-2} C_{2}}{8}-\frac{3 A_{0} B_{0} C_{0}}{4}-\frac{A_{0} B_{1} C_{-1}}{4} \\
& +\frac{A_{0} B_{2} C_{-2}}{8}-\frac{A_{1} B_{-1} C_{0}}{4}-\frac{A_{1} B_{-2} C_{1}}{4}-\frac{A_{1} B_{0} C_{-1}}{4} \\
& -\frac{A_{1} B_{1} C_{-2}}{4}-\frac{A_{2} B_{-1} C_{-1}}{4}+\frac{A_{2} B_{-2} C_{0}}{8}+\frac{A_{2} B_{0} C_{-2}}{8} .
\end{aligned}
$$

If $C$ gets a vev, then we can set $C_{0}=1$ and the other $C_{i}=0$. This then generates the bilinear operator

$\operatorname{tr}\left(A B C^{(0)}\right)=-\frac{A_{-1} B_{1}}{4}+\frac{A_{-2} B_{2}}{8}-\frac{3 A_{0} B_{0}}{4}-\frac{A_{1} B_{-1}}{4}+\frac{A_{2} B_{-2}}{8}$

This implies that the trilinear $\operatorname{tr}(A B C)$ is part of the $S O(3)$ chiral ring. Then the bilinear operator $\operatorname{Re}\left(A_{2} B_{-2}\right)-2 \operatorname{Re}\left(A_{1} B_{-1}\right)$ can be generated in terms of elements of the $S O(3)$ chiral ring. In combination with the bilinear (), all operators $\mathrm{O}_{1-3}$ can be generated.
The trilinear $\operatorname{tr}(A B C)$ also yields a trilinear in the $S O(2)$ fields if none of the tensors has a vev. This trilinear can be written as

$\operatorname{tr}(A B C)=-\frac{1}{4} \operatorname{Re}\left(A_{-1} B_{-1} C_{2}+A_{-1} B_{2} C_{-1}+A_{2} B_{-1} C_{-1}\right)+\ldots$

where the ellipses are combinations of $O_{1-3}$. Hence the completely symmetric trilinear is generated. To generate the other trilinears, we must consider a new $S O(3)$ invariant.

The next possibility is an invariant of degree $4 ; \operatorname{tr}(A B C D)$. However, we can narrow our search a little. The completely symmetrized trilinear has already been generated, and, so, we need to look for invariants where at least two tensors are antisymmetrized. There are exactly two such possibilities; (a) $\operatorname{tr}([A, B][C, D])$ and $(\mathrm{b}) \operatorname{tr}(A B C D)-\operatorname{tr}(C B A D)$. However the second can be shown to be zero for symmetric tensors.

We therefore include $\operatorname{tr}([A, B][C, D])$ in the Hilbert basis. We allow the field to get a vev (i.e. $D_{0}=1$ and the other $D_{i}=0$.). We then find

$\operatorname{tr}\left([A, B]\left[C, D^{(0)}\right]\right)=\frac{3}{2}\left(\operatorname{Re}\left(A_{-1} C_{-1} B_{2}\right)-\operatorname{Re}\left(B_{-1} C_{-1} A_{2}\right)\right)+\ldots$,

where the ellipses are combinations of $\mathrm{O}_{1-3}$. We thus generate the trilinear operator $\left(\operatorname{Re}\left(A_{-1} C_{-1} B_{2}\right)-\operatorname{Re}\left(B_{-1} C_{-1} A_{2}\right)\right)$. We also find that the $\operatorname{tr}([A, C][B, D])$ generates $\left(\operatorname{Re}\left(A_{-1} B_{-1} C_{2}\right)\right.$ $\left.-\operatorname{Re}\left(C_{-1} B_{-1} A_{2}\right)\right)$. Along with the completely symmetric trilinear, we have generated all trilinears from $\mathrm{O}_{4}$. This implies that the operator $\operatorname{tr}([A, B][C, D])$ is part of the $S O(3)$ chiral ring.

We now turn to the generation of all terms of the form $\mathrm{O}_{5}$. It is useful, however, to first work out what structures we are looking for. The $\mathrm{O}_{5}$ terms have the general form $\operatorname{Im}\left(A_{1} B_{-1}\right) \operatorname{Im}\left(C_{2} D_{-2}\right)$. There are other such forms related by a permutation acting on ABCD. There are 6 such independent permutations which are $\operatorname{Im}\left(A_{1} B_{-1}\right) \operatorname{Im}\left(C_{2} D_{-2}\right)$, $\operatorname{Im}\left(A_{1} C_{-1}\right) \operatorname{Im}\left(B_{2} D_{-2}\right)$, etc.

It is useful to separate these into combinations which are (anti)symmetric when we interchange the two pairs of fields. In the symmetric terms, we further isolate the expression which is antisymmetric under any pairwise exchange. These are

$$
\begin{aligned}
& \operatorname{Im}\left(A_{1} B_{-1}\right) \operatorname{Im}\left(C_{2} D_{-2}\right) \pm \operatorname{Im}\left(C_{1} D_{-1}\right) \operatorname{Im}\left(A_{2} B_{-2}\right) \\
& \operatorname{Im}\left(A_{1} C_{-1}\right) \operatorname{Im}\left(B_{2} D_{-2}\right) \pm \operatorname{Im}\left(B_{1} D_{-1}\right) \operatorname{Im}\left(A_{2} C_{-2}\right) \\
& \operatorname{Im}\left(A_{1} D_{-1}\right) \operatorname{Im}\left(B_{2} C_{-2}\right) \pm \operatorname{Im}\left(B_{1} C_{-1}\right) \operatorname{Im}\left(A_{2} D_{-2}\right)
\end{aligned}
$$

If no field gets a vev, the operator $\operatorname{tr}([A, B][C, D])$ generates an operator of the form $\mathrm{O}_{5}$ :

$$
\begin{aligned}
& \operatorname{tr}([A, B][C, D])=-\left(\operatorname{Im}\left(A_{1} B_{-1}\right) \operatorname{Im}\left(C_{2} D_{-2}\right)\right. \\
& \left.\quad+\operatorname{Im}\left(A_{2} B_{-2}\right) \operatorname{Im}\left(C_{1} D_{-1}\right)\right)+\frac{1}{2}\left(\operatorname{Im}\left(A_{1} D_{-1}\right) \operatorname{Im}\left(B_{2} C_{-2}\right)\right. \\
& \left.\quad+\operatorname{Im}\left(A_{2} D_{-2}\right) \operatorname{Im}\left(B_{1} C_{-1}\right)\right)-\frac{1}{2}\left(\operatorname{Im}\left(A_{1} C_{-1}\right) \operatorname{Im}\left(B_{2} D_{-2}\right)\right. \\
& \left.\quad+\operatorname{Im}\left(A_{2} C_{-2}\right) \operatorname{Im}\left(B_{1} D_{-1}\right)\right)+\ldots
\end{aligned}
$$

Similarly, we also have

$$
\begin{aligned}
& \operatorname{tr}([A, C][B, D])=-\left(\operatorname{Im}\left(A_{1} C_{-1}\right) \operatorname{Im}\left(B_{2} D_{-2}\right)\right. \\
& \left.\quad+\operatorname{Im}\left(A_{2} C_{-2}\right) \operatorname{Im}\left(B_{1} D_{-1}\right)\right)-\frac{1}{2}\left(\operatorname{Im}\left(A_{1} D_{-1}\right) \operatorname{Im}\left(B_{2} C_{-2}\right)\right. \\
& \left.\quad+\operatorname{Im}\left(A_{2} D_{-2}\right) \operatorname{Im}\left(B_{1} C_{-1}\right)\right)-\frac{1}{2}\left(\operatorname{Im}\left(A_{1} B_{-1}\right) \operatorname{Im}\left(C_{2} D_{-2}\right)\right. \\
& \left.\quad+\operatorname{Im}\left(A_{2} B_{-2}\right) \operatorname{Im}\left(C_{1} D_{-1}\right)\right)+\ldots
\end{aligned}
$$


Since we have

$$
\operatorname{tr}([A, B][C, D])-\operatorname{tr}([A, C][B, D])-\operatorname{tr}([A, D][C, B])=0
$$

the completely antisymmetrized structure cannot be generated. The operators $\operatorname{tr}([A, B][C, D]), \operatorname{tr}([A, C][B, D])$ thus generate two combinations of the $\mathrm{O}_{5}$ form which are symmetric under interchange of $(\mathrm{AB}),(\mathrm{CD})$, but not the fully antisymmetric combination. We therefore still need new $S O(3)$ invariants.

We must consider invariants of degree 5. These are of the form $\operatorname{tr}(A B C D E)$ where one field gets a vev, since we must generate a quadrilinear. We can take $E$ to be the field getting a vev; there are then 24 possible permutations of the remaining field in this invariant.

We first try to generate the completely antisymmetric (in $\mathrm{ABCD})$ structure. It is relatively easy to see that this requires the $S O(3)$ chiral ring to include the structure $\operatorname{tr}(\mathrm{ABCDE})$ where all five tensors are completely antisymmetrized.

We then generate the structures in which (AB), (CD) are antisymmetric, and where the structure is odd under pairwise interchange of $(\mathrm{AB}),(\mathrm{CD})$. The only possible nonzero candidate structure is

$$
\operatorname{tr}(A[C, D] B E)-\operatorname{tr}(C[A, B] D E)
$$

We include the last candidate as part of the chiral ring, and find its decomposition once $E$ gets a vev. This is

$$
\begin{aligned}
\operatorname{tr}\left(B E^{(0)} A[C, D]-D E^{(0)} C[A, B]\right)= & \frac{3}{2}\left(\operatorname{Im}\left(C_{1} D_{-1}\right) \operatorname{Im}\left(A_{2} B_{-2}\right)\right. \\
& \left.-\operatorname{Im}\left(C_{2} D_{-2}\right) \operatorname{Im}\left(A_{1} B_{-1}\right)\right) \\
\operatorname{tr}\left(C E^{(0)} B[D, A]-A E^{(0)} D[B, C]\right)= & \frac{3}{2}\left(\operatorname{Im}\left(A_{1} D_{-1}\right) \operatorname{Im}\left(B_{-2} C_{2}\right)\right. \\
& \left.-\operatorname{Im}\left(A_{2} D_{-2}\right) \operatorname{Im}\left(B_{-1} C_{1}\right)\right) \\
\operatorname{tr}\left(C E^{(0)} A[D, B]-B E^{(0)} D[A, C]\right)= & \frac{3}{2} \operatorname{Im}\left(A_{2} C_{-2}\right) \operatorname{Im}\left(B_{-1} D_{1}\right) \\
& \left.-\operatorname{Im}\left(A_{1} C_{-1}\right)\right) \operatorname{Im}\left(B_{-2} D_{2}\right)
\end{aligned}
$$

which, indeed, generates all the required $\mathrm{O}_{5}$ structures.

In summary, we have found the Hilbert basis for the $S O(3)$ group with symmetric tensors. These are:

$$
\begin{aligned}
& S_{1}=\operatorname{tr}(A B) \\
& S_{2}=\operatorname{tr}(A B C) \\
& S_{3}=\operatorname{tr}([A, B][C, D]) \\
& S_{4}=\operatorname{tr}(A B C D E) \quad \text { antisymmetrized in ABCDE } \\
& S_{5}=\operatorname{tr}(A[C, D] B E)-\operatorname{tr}(C[A, B] D E)
\end{aligned}
$$

In this note, we have presented a new approach to constructing the invariant tensors for theories with arbitrary representation content. We have applied this method to simple examples and shown that the known results are reproduced. We have also applied this method to a theory where the chiral ring was not known - a $S O(3)$ theory with symmetric tensors - and shown that we can extract the chiral ring by our methods.

While we have worked with relatively simple groups here we expect that our methods can be generalized straightforwardly to other groups and representations. In particular, it would be interesting to find invariant tensors for $S U(N)$ tensors with adjoint representations, which are interesting for the physics of strong interactions, and for exceptional groups, which are important for applications to dualities in supersymmetric theories.

\section{ACKNOWLEDGEMENTS}

This work was supported in part by NSF Grant No. PHY1620638.

\section{References}

[1] N. Seiberg, "Electric - magnetic duality in supersymmetric nonAbelian gauge theories," Nucl. Phys. B 435, 129 (1995) [hep-th/9411149].

[2] G. . 't Hooft, C. . Itzykson, A. . Jaffe, H. . Lehmann, P. K. Mitter, I. M. Singer and R. . Stora, "Recent Developments In Gauge Theories. Proceedings, Nato Advanced Study Institute, Cargese, France, August 26 - September 8, 1979," New York, Usa: Plenum (1980) 438 P. (Nato Advanced Study Institutes Series: Series B, Physics, 59).

[3] D. Kutasov, A. Schwimmer and N. Seiberg, "Chiral Rings, Singularity Theory and Electric-Magnetic Duality," Nucl. Phys. B 459, 455 (1996) [hep-th/9510222].

[4] P. Brax, C. Grojean and C. A. Savoy, "Anomaly matching and syzygies in $\mathrm{N}=1$ gauge theories," Nucl. Phys. B 561, 77 (1999) [hep-ph/9808345].

[5] P. Pouliot, "Molien function for duality," JHEP 9901, 021 (1999) [hep-th/9812015].

[6] H. Weyl, "The Classical Groups, Their Invariants and Representations," Princeton University Press, 1946.

[7] W. Fulton and J. Harris, "Representation Theory, A First Course," Springer-Verlag, 1991.

[8] P. Olver, "Classical Invariant Theory," London Mathematical Society Student Texts \#44, 1999.

[9] J. Dieudonne and J. Carrell, "Invariant Theory, Old and New," Academic Press, 1971.

[10] R. Howe, "Perspectives on Invariant Theory," The Schur Lectures (1992), Israel Mathematical Conference Proceedings, 1995.

[11] G. Gurevich, "Foundations of the Theory of Algebraic Invariants," P. Noordhoff - Groningen, The Netherlands, 1964.

[12] G. Schwarz, "Invariant theory of $G_{2}$," Bull. Am. Math. Soc. 9, 335 (1983).

[13] G. Schwarz, "Invariant theory of $G_{2}$ and Spin 7 ," Comment. Math. Helvetici 63, 624 (1988).

[14] G. Schwarz, "Representations of Simple Lie Groups with a Free Module of Covariants," Inventiones Math. 50, 1 (1978).

[15] G. Schwarz, "Representations of Simple Lie Groups with Regular Rings of Invariants," Inventiones Math. 49, 167 (1978).

[16] V. Popov, "Syzygies in the Theory of Invariants," Math. USSR Izvestiya 22, 507 (1984).

[17] Y. Gufan, Al. V. Popov, G. Sartori, V. Talamini, G. Valente and E. Vinberg, "Geometric Invariant Theory Approach to the Determination of Ground States D-wave Condensates in Isotropic Space," Jour. Math. Phys. 42, 1533 (2001).

[18] P. Pouliot, J. Phys. A 34, 8631 (2001) doi:10.1088/03054470/34/41/317 [hep-th/0107151]. 
[19] F. Buccella, J. P. Derendinger, S. Ferrara and C. A. Savoy, "Patterns Of Symmetry Breaking In Supersymmetric Gauge Theories," Phys. Lett. B 115, 375 (1982).

[20] C. Procesi and G. W. Schwarz, "The Geometry Of Orbit Spaces And Gauge Symmetry Breaking In Supersymmetric Gauge Theories," Phys. Lett. B 161 (1985) 117.

[21] R. Gatto and G. Sartori, "Consequences Of The Complex Character Of The Internal Symmetry In Supersymmetric Theories," Commun. Math. Phys. 109, 327 (1987).

[22] M. A. Luty and W. I. Taylor, "Varieties of vacua in classical supersymmetric gauge theories," Phys. Rev. D 53, 3399 (1996) [hep-th/9506098].

[23] T. Gherghetta, C. Kolda and S. P. Martin, "Flat directions in the scalar potential of the supersymmetric standard model," Nucl. Phys. B 468, 37 (1996) [hep-ph/9510370].

[24] P. Brax and C. A. Savoy, "Supersymmetric flat directions and analytic gauge invariants," hep-th/0104077.

[25] C. Csaki and H. Murayama, "Discrete anomaly matching," Nucl. Phys. B 515, 114 (1998) [hep-th/9710105].

[26] P. C. Argyres, M. Ronen Plesser and N. Seiberg, "The Moduli Space of N=2 SUSY QCD and Duality in N=1 SUSY QCD," Nucl. Phys. B 471, 159 (1996) [hep-th/9603042].

[27] M. Berkooz, "A Comment on Non-Chiral Operators in SQCD and its Dual," Nucl. Phys. B 466, 75 (1996) [hepth/9512024].

[28] M. van Leeuwen, A. Cohen and B. Lisser, "LiE Manual," version 2.1, Amsterdam, The Netherlands, 1996. 